\title{
Assessment of a Database Management System in a Mammogram Unit of a Radiologic Department in Fann Teaching Hospital (Senegal)
}

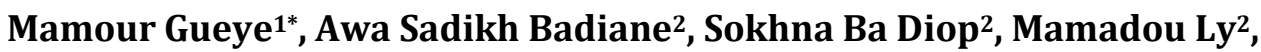 \\ Mame Diarra Ndiaye Gueye, Abdoulaye Dione Diop², Abdoulaye Ndoye Diop², \\ Marame Fall²
}

\author{
${ }^{1}$ Gynecologic and Obstetric Clinic, Aristide Le Dantec Teaching Hospital, Dakar, Senegal \\ ${ }^{2}$ Department or Radiology, Fann Teaching Hospital, Dakar, Senegal \\ Email: *mamourmb@yahoo.fr
}

How to cite this paper: Gueye, M., Badiane, A.S., Ba Diop, S., Ly, M., Ndiaye Gueye, M.D., Dione Diop, A., Ndoye Diop, A. and Fall, M. (2017) Assessment of a Database Management System in a Mammogram Unit of a Radiologic Department in Fann Teaching Hospital (Senegal). International Journal of Medical Physics, Clinical Engineering and Radiation Oncology, 6, 401-409.

https://doi.org/10.4236/ijmpcero.2017.64036

Received: September 25, 2017

Accepted: October 31, 2017

Published: November 3, 2017

Copyright (c) 2017 by authors and Scientific Research Publishing Inc. This work is licensed under the Creative Commons Attribution International License (CC BY 4.0).

http://creativecommons.org/licenses/by/4.0/

\begin{abstract}
Objectives: To evaluate a medical data management system of a mammogram unit in a department of Radiology. Methods: This is a qualitative and quantitative assessment study in Fann Teaching Hospital between April 2014 and June 2015 one year after its implementation. The quantitative component consisted of the audit of the database to determine the socio-demographic characteristics of patients and the results of mammograms. The qualitative component assessed users' experience. For analysis, quantitative data were extracted and transferred to Microsoft Excel. For scale variables, we calculated the averages and extremes. For qualitative variables, we established percentages. Results: During the study period, 433 patients underwent mammograms. The average age of patients was 48 years. The completion rate maintained above $85 \%$ was below $26 \%$ in the first two months of use. As to the completeness given examinations, it was still above $83 \%$. The results of mammogram examinations were normal in the majority of cases: $96 \%$ for the right breast and $95.2 \%$ for the left breast. All users had a favourable opinion about the database. The reasons were better work organization, comprehensiveness, accessibility and standardization of information about the patient and especially the immediate availability of statistics. For $60 \%$ of these health professionals, complaints related to the use of the software were the time-consuming of filling data. Conclusion: This study mainly describes the perception of health professionals on the computerization of radiological examinations. It offers some advantages, proposes improvements and opens
\end{abstract}


avenues for reflection on the globalization of the computerization of patient records in Radiology.

\section{Keywords}

Electronic Medical Records, Database, Mammogram, Radiology, Dakar

\section{Introduction}

In Computer Science, a database management system (DBMS) is a system software for storing and sharing information in a database, guaranteeing the quality, durability and confidentiality of information while hiding the complexity of operations. DBMS is intermediate software between users and databases.

Today, computing is not only a valuable aid for the medical profession, it is at the heart of modern medicine, a medicine that requires information sharing between health system stakeholders and the patient in order to improve care, coordination and continuity of care.

While computerization of the medical record is increasingly evolving in western countries, it is slow to start in Africa. From a chronological point of view, several decades separate us. In 2006, an informal survey carried out in fifteen Francophone African countries through the coordination of the Telemedicine network in French-speaking Africa confirmed that there was no hospital information system integrating the management of medical information [1].

At present, in our country, hospitals with this tool have the departmental computerization model, i.e., each service manages its own database as this is the case in the medical imaging department of Fann Teaching Hospital in Dakar the capital of Senegal where a mammography report management software has been implemented.

The general objective of this study was to evaluate this system of management of automated mammography reports in a Radiology department.

\section{Methodology}

This study was conducted at the Department of Radiology at Fann Teaching Hospital in Dakar Senegal. It evaluated a Database Management System (DBMS) in a Radiology unit between April 2014 and June 2015. Prior to April 2014, mammography reports were entered using Microsoft Word 2007 software. For each patient, the previous report was duplicated and the report number incremented manually by one. The monthly reports were tedious and carried out by an executive assistant over several days.

A medical developer of medical data management systems initiated this DBMS. It would manage mammography reports, the recording of patient demographics and the automated management of monthly, quarterly and annual reports. 
The DBMS was designed using the Filemaker Pro software. FileMaker Pro is a database management software developed by an Apple subsidiary called FileMaker Inc. It runs on Mac OS X and Windows, either in peer-to-peer network or in client-server mode with the "FileMaker Server" module.

Entering into the database is possible by using an account name and a password.

The use is then intuitive and information are entered via successive windows, first administrative, socio-demographic and then medical in connection with the examination in progress. The first two information orders are recorded for an epidemiological purpose, but only information related to the current examination were included in the mammography report.

Several buttons allow creating a new record (new patient), a new examination (new mammogram). Indeed, the same patient seen to another date will benefit from another mammogram using its already registered demographic characteristics. The other buttons allow the establishment of the billing grid, display of statistics of monthly activities, synopsis in the form of a register, etc. Previous and completed mammograms also appear on the home page.

Mammography data included symmetry, breast density, opacity, mammary distortion, asymmetric focal density, micro calcifications, axillary nodes as well as the appearance of the skin. Each abnormality is described and its localisation specified.

To optimize the filling, all the boxes not filled appear in a pink background. The fill changed the display to a light background. There are three free-writing sections at the bottom of the page.

Conclusion is written in free text and then categorized according to the classification of the American College of Radiology (ACR).

Complementary analyses relate to statistics to give a visibility of the activity.

Half-day training was organized for all users.

After one year of use, an assessment was necessary. This is the objective of this study. This study had a quantitative component and a qualitative component. The quantitative aspect consisted of recording the number of patients recorded during the study period, their socio-demographic characteristics, the reasons and the methods of mammography and the results of exams. In addition, the completeness of the data recording was assessed and monitored over time. The qualitative component assessed users' experience.

The quantitative data were extracted and transferred to Microsoft Excel 2010 software for analysis. For scale variables, we calculated averages and extremes. For the qualitative variables, we had established percentages.

\section{Results}

\subsection{Sociodemographic Data}

During the study period, 433 patients underwent mammograms. The average age of patients was 48 years. Patients' sociodemographic characteristics are shown in Table 1 . 
Table 1. Sociodemographic characteristics of patients.

\begin{tabular}{ccc}
\hline Variables & Number & Percentage (\%) \\
\hline Age (years) & 74 & 17.1 \\
\hline $19-30$ & 308 & 71.1 \\
$31-59$ & 51 & 11.8 \\
$>59$ & & 15.5 \\
\hline Number of pregnancy & 67 & 84.5 \\
\hline 0 & 366 & 22.8 \\
\hline and more & & 77.2 \\
\hline Number of delivery & 99 & 12.5 \\
\hline 1 and more & 334 & 87.5 \\
\hline History family of breast cancer & & \\
\hline Yes & 54 & \\
No & 379 & \\
\hline
\end{tabular}

\subsection{Completeness of Information}

Completeness was assessed on the filling of the following 14 parameters during registration: date of first contact, first name, second name, age, address, telephone, age at first menstruation, number of pregnancy, parity, number of live children, abortions, age at first pregnancy, cumulative breastfeeding duration and history of breast cancer.

During the first two months of registration, the completeness rate was below $26 \%$. It had grown up rapidly and remained above $85 \%$ as shown in Figure 1. Activities were interrupted in June 2014.

Concerning the completeness of the data of the mammographic examination, it was satisfactory and has always maintained above $83 \%$ (Figure 2).

\subsection{Socio-Demographic Data of the Patients}

From the beginning of April to the end of June 2015, 433 patients performed a mammogram.

The average age of patients was 48 years with extremes of 19 and 81 years.

The results of the examination were normal in the majority of cases (96\% for the right breast and $95.2 \%$ for the left breast).

\subsection{Qualitative Results}

The qualitative component of our study was based on a questionnaire submitted to users of the database and individual interviews.

All respondents, including a physician, three executive assistants and a radiology manipulator were interviewed. 


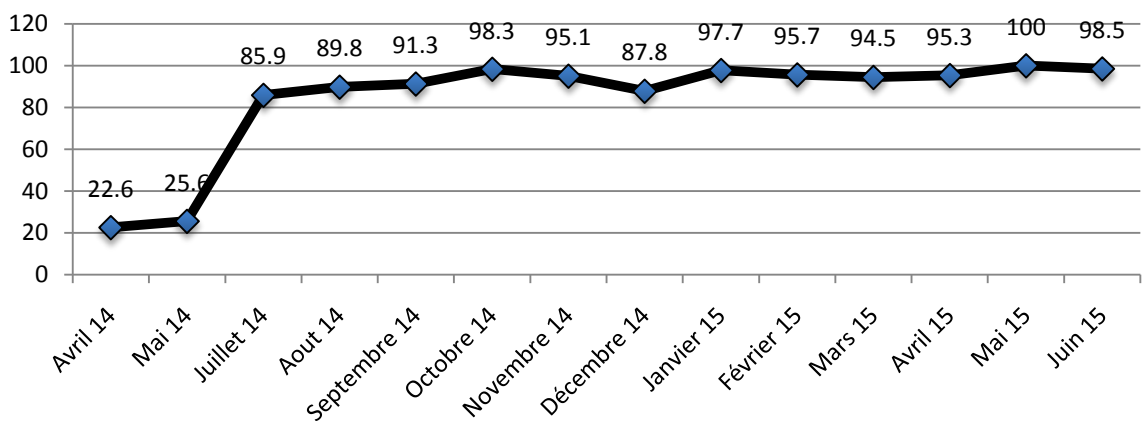

Figure 1. Monthly evolution of record completeness.

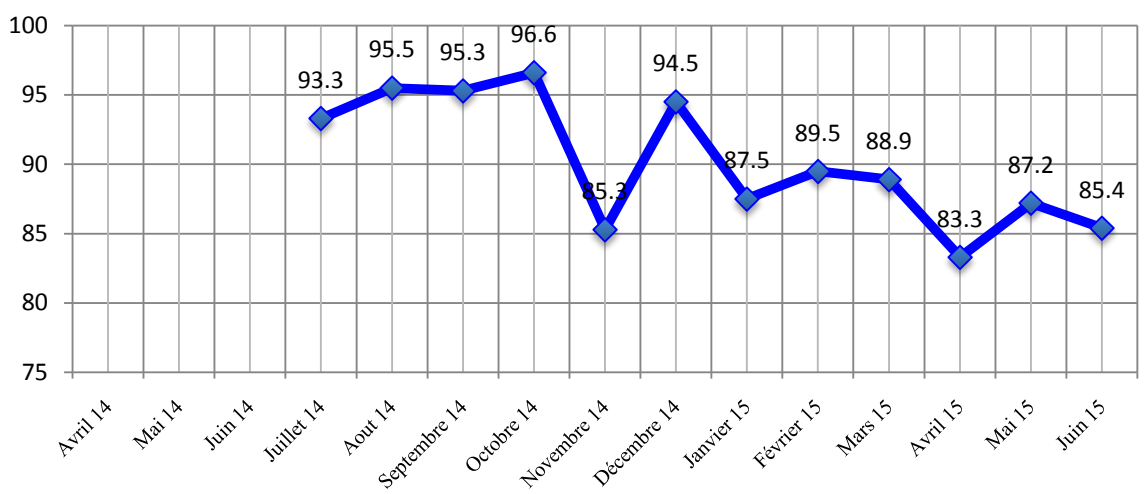

Figure 2. Monthly evolution of the completeness of the mammographic examination data.

The entire population had so-called "basic" computer skills and felt comfortable with the professional use of computers. Out of these five interviewees, a doctor and an executive assistant were trained in the use of the software and were satisfied with the training. The remaining three had been initiated "in the pile" by the trained management assistant.

There was unanimous support for the computerization of mammography reports, with the main reasons given for better organization of the work, completeness, accessibility and standardization of the information collected on the patient and immediate availability statistics.

As for its use, the points to improve raised belonged to the speeches of the assistant directors $(60 \%)$ which emphasized the time-consuming character of the filling of the interfaces.

$20 \%$ of respondents felt that the computerization of mammography records had no impact on the care of patients (Figure 3). Sixty per cent of them saw a positive aspect: saving time in known patients, real-time data capture, good archiving and quick comparison of previous exams, simplifying results around a common language.

Eighty per cent said that computerization had an impact on the organization of the department. One person claimed that computerization had introduced self-discipline and made mammography records accessible, three people referred to better archiving and automatic and more reliable statistics. 


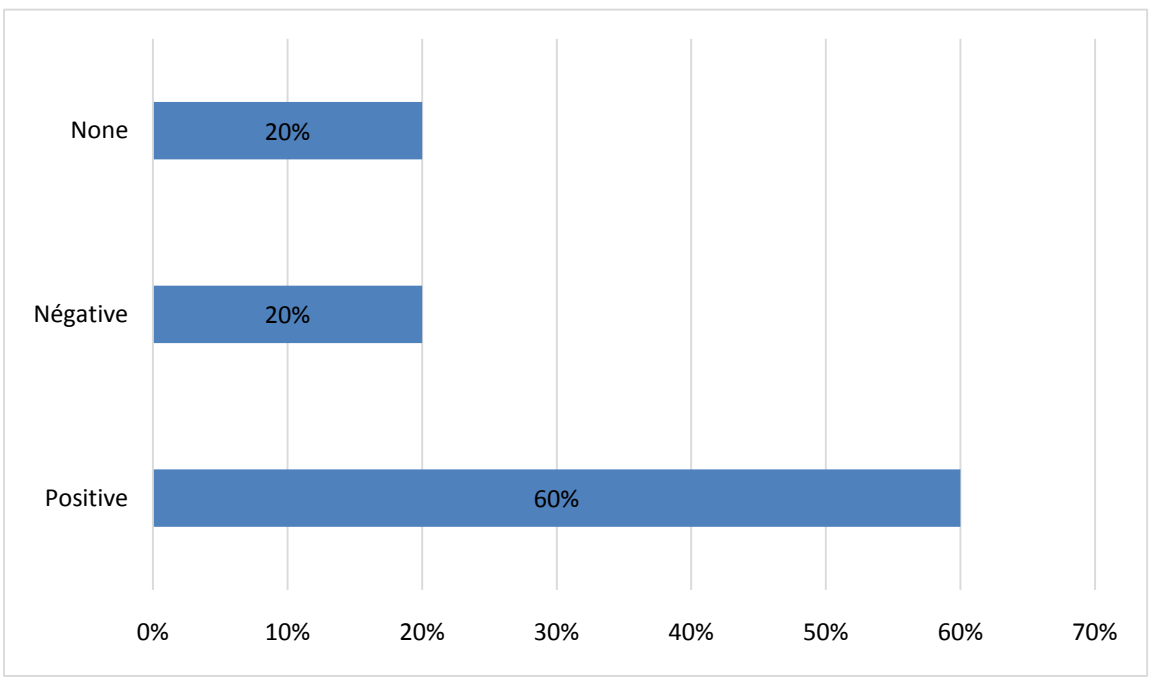

Figure 3. Distribution of users according to their opinion on the impact of the software on the care of patients.

\section{Discussion}

\subsection{Strengths of the Study}

This study enters into the small circle of work on the computerization of patient records in French-speaking Africa and deals with an issue that could improve the control of medical activities and provide some solutions to public health needs by setting up reliable means of assessing and analysing the health status of populations.

\subsection{Limitations of the Study}

The limitations of our study are attributable to the limited nature of the workforce studied, which has reservations about the probability that it reflects the overall opinion of a department.

It is therefore partly a qualitative study, which does not pretend to be representative but rather informative of the personal opinion of professionals on the computerization of the files of imaging exams.

Further studies should be carried out including an in-depth study of the subject by a more exhaustive collection of the opinion of professionals and, within a broader framework, integration into the study of the patients' position in this innovation.

\subsection{Advantages of Electronic Medical Records}

Through the medical record, large amounts of information are generated daily by medical activities. This information is recorded in essentially textual documents, images and files whose mode of access and exploitation are mainly done manually and therefore difficult to reuse and share because of their structural heterogeneity.

To keep these millions of information collected, processed, analysed and ex- 
changed, databases are required, which necessarily implies computerization of the patient's record.

Benefits of Electronic medical records include clinical, organizational, and societal outcomes.

\subsection{Comparison with Other Medical Data Management Systems}

In terms of comparison with other database management systems, the majority of the subjects deal with medical specialties, but there are very few studies devoted to the exclusive domain of imaging with an individualization of the Radiologic file.

At the end of this evaluation, we found the Software users to have a favourable perception to the computerization of the mammographic examination files. Overall, they noted an improvement in the quality of the work and had identified its usefulness from the individual as well as the collective point of view, but with some reluctance regarding the time devoted to the typing, the only negative element drawn from this study. It had resulted in changes in professional practices, most of which were rather positive: automatic statistical development, accessibility of files, better archiving, quick comparison of exams, etc. Overall, these results were consistent with those found in the literature.

The University Hospital of Reims in France, which began the computerization of patients' files in 1986, attempted in 2004 to describe its experience on the Relational File of Events and Medical Acts application deployed since 2001 [2]. Benefits listed included data accessibility, facilitation of communication among professionals, statistical analysis facilitated by computerized data management, elimination of certain administrative tasks performed by healthcare providers, reports and statistics by executive assistants-as well as coding and invoicing of acts in real time. Security and privacy had also been described as key points in the computerized medical record, as well as improved archiving of records to ensure data sustainability [2]. We found this analogy in our study. In conclusion, the results of this work were similar to the qualitative results of our study.

The computerization of patient files seemed to be welcomed by users despite certain limitations and concerns about its use.

Regarding the completeness of information, interviews with management assistants attempted to identify the reasons of incompleteness of information during the first two months of the Software use. Administrative staff had confessed a lack of understanding of the software's early functionality and the loss of time caused by lack of experience. Indeed, the results of the survey revealed that only one administrative staff member included in the study had been trained in the software during its implementation. For the others, it was a cascade. An already trained agent initiated most users. As for the $100 \%$ bar, it remained virtually out of reach since the deployment of the system. This non-filling was explained by the social context of patients' illiteracy. Management assistants reported that patients were unaware of certain administrative information such as age, but most often it was the gynaecological and obstetric antecedents, notably the menarches 
that were not reported.

The capture of medical data, although satisfactory, was also incomplete but the reasons for this situation had not been specified. Examination results being reported on paper and subsequently typed by administrative staff, the incompleteness of the examination data may be due to omissions in the computerized transcription of the results by the assistants and more likely by omissions on the part of the operators in charge of the examinations because, as we mentioned during its presentation, the software had a help function for the optimization of the filling.

This incompleteness of computerized records was not specific to our study. It was also found in literature.

The qualitative assessment of the information available in the Cinz@n database also showed an incompleteness of medico-administrative information, also explained in part by the fact that some patients did not know their age or other administrative information [1].

\subsection{Outlook and Improvements}

Despite the enthusiasm of health professionals for the software, limitations to its use persisted. This was related to the non-completion of certain information justified by "the too high number of elements to be fulfilled in relation to before" and "the lack of understanding of the functionalities of the software". As suggested by some studies, regular evaluation and training could help to overcome these limitations by identifying the dysfunctions encountered and implementing remedial measures. In the long term, it will be necessary to consider the recruitment of qualified personnel in the field of medical informatics [1] [3] [4] [5].

Nevertheless, at the end of this evaluation we can say without any doubt that the users have found a good compromise between the benefits and the disadvantages of the software so that the prospect could turn to an extension to the other imaging exams.

A report application similar to the software has been noted in the literature but which associated remarkable differences. This software included a system for verifying the concordance between the selected description and the American College of Radiology (ACR) BI-RADS (Breast Imaging-Reporting and Data System) classification, thus signalling discrepancies by alerts. In addition, at the end of the description of the examination, behaviour to keep according to the classification is displayed by default and a textual report is generated automatically and both remain modifiable leaving a traceability of the modification. Similarly, if a previous balance sheet is mentioned on the home page, a comparison is requested. In addition, help bubbles appear to give definitions and/or examples as well as lesion localization schemes [6].

These listed items, technically feasible in the short term, could be major assets in the management of patient records in imaging and the systematization of reports to avoid transcription errors. 


\section{Conclusion}

More than a quantitative study, this evaluation study mainly describes the perception of health professionals on the computerization of radiological examinations. It offers certain advantages, proposes improvements and opens up avenues for reflection on the globalization of the computerization of patient files in Radiology.

\section{References}

[1] Bagayoko, C.O. (2010) Miseen place d'un Systèmed' Information Hospitalieren Afrique Francophone: Cinz@n, étude et validation du modèle au Mali. [Establishment of a Hospital Information System in Francophone Africa: Cinz@n, Study and Validation of the Model in Mali.] Thèse Santé Publique et Recherche Clinique, Université de la Méditerranée, Aix-Marseille II.

[2] Marcus, C., Gomes, H., Hornoy, P., Roesch, S., Husson, R. and Menanteau, B. (2004) Dossier patient informatisé: rêVeouréalité. [Computerized Patient Record: Dream or Reality.] Journal de radiologie, 85, 1614. https://doi.org/10.1016/S0221-0363(04)78063-4

[3] Boyer, L., Renaud, M.H., Baumstarck-Barrau, K., Fieschi, M. and Samuelian, J.C. (2010) Establishment of an Electronic Medical Record in a Psychiatric Hospital: Evolution of Professionals' Perceptions. L'Encéphale, 36, 236-241. https://doi.org/10.1016/S0221-0363(04)78063-4

[4] Rose, A.F., Schnipper, J.L. and Park, E.R. (2005) Using Qualitative Studies to Improve the Usability of an EMR. Journal of Biomedical Informatics, 38, 51-60. https://doi.org/10.1016/j.jbi.2004.11.006

[5] Roukema, J., Los, R.K., Bleeker, S.E., Van Ginneken, A.M., Van Der Lei, J. and Moll, H.A. (2006) Paper vs Computer: Feasibility of an Electronic Medical Record in General Pediatrics. Pediatrics, 117, 15-21. https://doi.org/10.1542/peds.2004-2741

[6] Jencikova, M.E., Tardivon, A., Rizand, P. and Neuenschwander, S. (2004) Une application de compte rendu mammographique informatisé. [A Computerized Mammographic Report Application.] Journal de Radiologie, 85, 1613. 